\title{
Spektroskopische Untersuchungen zur fluorometrischen Steroidanalyse
}

\author{
Von \\ R. Abraham und Hj. Staudinger \\ Aus dem Plyssiologisch-Chemischen Institut der Justus-Liebig-Universität Gießen (Dir.: Prof. Dr. Hj. Staudinger) \\ Herrn Prof. Dr. Fritz KRöHNKE zum 60. Geburtstag gewidmet
}

(Der Schriftleitung zugegangen am 5. Juli 1963)

\begin{abstract}
In der vorliegenden Arbeit wurde die Fluoreszenz der Steroide in starker Mineralsäure näher untersucht. - Als Grundlage für eine spezifische Bestimmung einzelner Steroidhormone und ihrer Stoffwechselprodukte wurden die Anregungs- und Fluoreszenzspektren von 21 Steroiden mit dem Zeiss'schen Doppelmonochromatorgerät aufgenommen und zur Festlegung reproduzierbarer fluoreszenzspektroskopischer Daten Standardbedingungen für die Etzeugung der Fluoreszenzchromogene ausgearbeitet. Die Auswahl der Substanzen erfolgte überwiegend nach strukturchemischen Gesichtspunkten. Die spektroskopischen Daten eignen sich zur İdentifizierung von Steroiden, besonders in Verbindung mit den unter gleichen Bedingungen aufnehmbaren Absorptionsspektren im sicht= baren und UV-Bereich. Auf Grund vergleichender Untersuchungen konnten folgende Aufschlüsse über die strukturellen Voraussetzungen für die Fluoreszenz der Steroide gewonnen werden:

Alle Sauerstoff-Funktionen im Steroidmolekül können, wenn auch verschieden stark, als Fluoresżenzchromophore wirken. Ferner wird das Fluoreszenzvermögen der einzelnen Steroide stark durch den Typ des Steroidgerüstes bestimmt. Weitere Untersuchungen dienten der Aufklärung der Struktur der Steroid-Fluoreszenzchromogene in konz. Schwefelsäure. Bei Zusatz von aromatischen Carbonylverbindungen, die in konz. Schwefelsäure Oniumsalze bilden, treten Veränderungen in Lage und Intensität der Spektren der Fluoreszenzchromogene auf, welche als eine Assoziation mit den Carbonylverbindungen gedeutet werden. Somit ist eine Oniumsalzstruktur der Fluoreszenzchromogene von Steroiden wahrscheinlich. Hierfür werden verschiedene Formulierungen vorgeschlagen und im Hinblick auf den Zustand der Fluoreszenzchromogene in Lösung diskutiert.
\end{abstract}

The fluorescence of steroids in strong mineral acids was studied. As a basis for the specific determination of individual steroid hormones and their metabolites, the excitation and fluorescence spectra of 21 steroids were recorded with a Zeiss double monochromator. Conditions for the production of fluorescence chromogens were standardised to ensure reproducible fluorescence spectroscopic data. The choice of substance was determined chiefly by structural chemical considerations. The spectroscopic data are suitable for identification, especially when supplemented by visible and UV absorption spectra made under the same conditions. On the basis of comparative studies, the following conclusions were drawn concerning the structural requirements for steroid fluorescence:

All oxygen functions in the steroid molecule can act as fluorescence chromophores, albeit of varying intensity. Also, the fluorescence of individual steroids is strongly influenced by the type of steroid skeleton.

Further investigations served to explain the structure of steroid fluorescence chromogens in conc. sulphuric acid. Addition of aromatic carbonyl compounds, which form onium salts in conc. sulphuric acid, caused a change in the position and intensity of the spectrà of the fluorescence chromogens. This indicates an association with the carbonyl compounds. Thus the fluorescence chromogens of steroids probably have an onium salt structure. Various formulations of this are suggested and discussed with respect to the state of the fluorescence chromogens in solution.

Die fluorometrische Analyse von Steroidhormonen stellt im Prinzip eine spektrophotometrische Bestimmung der in starken Mineralsäuren entstehenden Fluoreszenzcbromogene dar. Das Verfahren kann als eine der empfindlichsten Mikromethoden der chemischen Steroidbestimmung bezeichnet werden, die sich überdies auf eine große Anzahl einzelner Steroide anwenden läßt (1-7). - Es liegt bereits eine Reihe von Untersuchungen vor, deren Ziel es war, die bei der Reaktion mit starken Mineralsäuren entstehenden Fluoreszenzchromogene der Steroide hinsichtlich der Fluoreszenzmaxima und der Reaktionsbedingungen (Acidität, Zeit, Temperatur) näher zu definieren (8-10). Die Ergebnisse dieser Arbeiten, die für fluorometrische Steroidbestimmungen sehr wertvoll geworden sind, lassen noch drei wichtige Fragen offen, deren Beantwortung für die weitere methodische Grundlegung von Bedeutung sind:
1. Besitzen die Fluoreszenzchromogene der Steroide neben einem individuellen Fluoreszenzspektrum ein für verschiedene Steroide spezifisches Anregungsspektrum, das bei der Entwicklung möglichst spezifischer fluorometrischer Bestimmungsmethoden für die einzelnen Steroide berücksichtigt werden muß?

2. Welche Beziehungen bestehen zwischen Fluoreszenzintensität und dem Anregungs- bzw. Fluoreszenzspektrum einerseits und der chemischen Konstitution des Steroids andererseits?

3. Welche chemische Struktur kann für diese Chromogene in konz. Schwefelsäure angenommen werden?

\section{Methodik}

\section{Fluoreszenzspektroskopische Messungen}

Die Anregungs- und Fluoreszenzspektren wurden mit einem Spektralffuorometer mit zwei Monochromatoren der Fa. C. Zeiss, Obetkochen, aufgenommen. Das Gerät stellt eine Kombination 
des Spektralphotometers PMQ II mit Fluoreszenzansatz ZMF 4 und einer Xenonlampe mit vorgeschaltetem Monochromator M 4 Q Il als Lichtquelle für das Anregungslicht dar. Die Xenonlampe wird über ein Anschlußgerät, das kontinuicrlich regelbare Gleichspannungen licfert, versorgt. Zum Ausgleich der Netzspannungsschwankungen, gegen welche der Xenonlichtbogen schr empfindlich ist, wird die Netzspannung mit einem leistungsfähigen Stabilisator konstant gehalten. Die Verstärkung des Photostroms im Anzeigegerät wurde durch Einbau eines geeigneten Widerstandes um den Faktor 3,3 gegenüber der handelsüblichen Ausführung erhöht.

Vor jeder Messung wurde das Gerät mit dem beigegebenen Fluoreszenzstandard geeicht. Die Aufnahme der Spektren exfolgte ohne Registriereinrichtung durch Punkt für Punkt-Messung, indem bei konstanter Anregungswellenlänge das Fluoreszenzspektrum im Sekundärmonochromator und anschließend durch Einstellung auf das so ermittelte Fluoreszenzmaximum das Anregungsspektrum im Primärmonochromator durchgemessen wurde. Bei allen Messungen betrug die Spaltbreite am Primärmonochromator $0,5 \mathrm{~mm}$ und am Sekundärmonochromator $0,1 \mathrm{~mm}$. Dies entspricht einer effektiven Bandbreite von $6-7 \mathrm{~m} \mu$ (für Anregungsmaxima zwischen 380 und $480 \mathrm{~m} \mu$ ) bzw. etwa $4 \mathrm{~m} \mu$ (für Fluoreszenzmaxima um $530 \mathrm{~m} \mu$ ). Zum Schutz des Primärmonochromators vor der Strahlenbelastung durch die Xenonlampe wird üblicherweise zwischen Xenonlampe und Primärmonochromator eine Wasserküvette gebracht, in welcher sich zusätzlich ein UV-durchlässiges Filter befindet (Absorptionsmaximum bei $395 \mathrm{~m} \mu$ ). Die Lage der Anregungsmaxima und der Verlauf der Anregungskurven wird außer durch die Intensitätsverteilung der Lichtquelle noch von den in der gesamten Meßanordnung möglichen Filterwirkungen stark beeinflußt. Daher wurden die Messungen an den reinen Steroiden ohne das „Schutzfilter" durchgeführt. Dabei muß darauf geachtet werden, da $\beta$ nicht länger als 10 Minuten gemessen wird, da sonst der Primärmonochromator geschädigt wird. Unter diesen Bedingungen wurden die in Tabelle 1 angegebenen Daten gefunden. Bei der Untersuchung der Spektren in Gegenwart von verschiedenen Carbonylverbindungen hat sich die Benutzung des UV-durchlässigen Filters als notwendig erwiesen, da die in dem Bereich $400-425 \mathrm{~m} \mu$ zusätzlich auftretenden Anregungsmaxima (Tabelle 2) nur mit der durch das Filter veränderten spektralen Intensitätsverteilung der Xenonlampe in Erscheinung treten. Der Einfluß der veränderten Intensitätsverteilungen auf Lage und Verlauf der Anregungskurven wird bei der Diskussion der Ergebnisse behandelt.

\section{Substanzen}

Die für die Untersuchung herangezogenen Steroidpräparate stammten aus dem Handel oder wurden von verschiedenen Forschungslaboratorien zur Verfügung gestellt ${ }^{1}$ ). Die Präparate waren als chemisch rein deklariert und wurden als solche verwendet. Steroidproben aus verschiedenen Quellen lieferten innerhalb der Reproduzierbarkeitsgrenze die gleichen spektroskopischen Daten, auch wenn die Dünnschichtchromatographie Verunreinigungen von 2-5\% erkennen ließ. Am Beispiel der Verbindungen Reichstein's Substanz S, $\alpha$-Cortolol, Corticosteron und Cortisol konnte gezeigt werden, daß die fluoreszenzspektroskopischen Daten nach dünnschichtchromatographischer Reinigung nicht vetändert werden.

\section{Schwefelsäure - Standardmetbode}

Beșonders wichtig für die Aufnahme reproduzierbarer Spektren ist die Standardisierung der Reaktionsbedingungen, da Lage und Intensität der Fluoreszenz- und Anregungsmaxima von zahlreichen

1) Für die Uberlassung von Steroidpräparaten danken wir:

Herrn Dr. Peterson, The Upjohn Company, Kalamazoo

Herrn Priv.-Doz. Dr. HübenER $†$, Frankfurt/M.

Fa. Schering AG., Berlin-West.

Herrn Prof. Dr. T. Reichstern, Basel.

Herrn Dr. Wertstenn, Basel.
Faktoren, wie z. B. Temperatur, Reaktionsdauer, Wassérgchalt der Mineralsäure, abhängen. Wir haben auf Grund von Vorversuchen eine für alle der untersuchten Steroide brauchbare Standardmethode entwickelt, die möglicherweise nicht für alle verwendeten Steroide optimal ist, dafür jedoch bei allen Steroiden unter einheitlichen Bedingungen gut meßbare Intensitäten liefert, so daß vergleichende Untersuchungen über das Fluoreszenzvermögen einzelner Steroide und Steroidgruppen möglich werden. Wir verwendeten folgende Methodik:

$0,2 \mu \mathrm{Mol}$ des Steroids werden in methanolischer Lösung (100 mg-proz.) in ein Schliffröhrchen pipettiert, das Lösungsmittel abgedampft und $3,0 \mathrm{ml}$ konz. Schwefelsäure („Merck“, zur Analyse, $d=1,84$, gut verschlossen aufbewahrt) zugegeben. Dann erhitzt man das Röhrchen unter gutem Verschluß in einem Wasserbad von $90^{\circ} 10 \mathrm{Min}$. im Dunkeln und kühlt unter der Wasserleitung ab. Nach weiteren $15 \mathrm{Min}$. wird der Ansatz in eine $1 \mathrm{~cm}$-Quarzküvette mit poliertem Boden eingefüllt und mit der Aufnahme der Spektren begonnen. Zuerst werden die Anregungsspektren, dann die Fluoreszenzspektren gemessen.

\section{Ergebnisse}

\section{Optische Daten der Fluoresqenzclbromogene in konz. Schivefelsäure}

In Tabelle 1 sind die Anregungs- und Fluoreszenzmaxima dargestellt, wie sie mit der „SchwefelsäureStandardmethode“ erhalten wurden. Die Wellenlängenangaben beziehen sich jeweils auf ein Spektrum, dessen Maxima ihrer Lage nach Mittelwerte zwischen den bei der Prüfung der Reproduzierbarkeit beobachteten Extremwerten darstellen. Dieses Spektrum wurde aus mindestens 3 Aufnahmen konstruiert. Zum Vergleich der Fluoreszenzfähigkeiten der verschiedenen Steroidmoleküle wurde die unter den Bedingungen der Standardmethode gemessene Intensität mit angegeben, und zwar in Skalenteilen des Galvanometer-Anzeigegeräts. Lichtquelle: Xenonlampe XBO $450 \mathrm{~W}$ ohne UV-durchlässiges Filter.

\section{Veränderungen in den Anregungsspektren der Fluores- zenzchromogene in Gegenwart von Vanillin}

In Tabelle 2 werden die in Gegenwart von Vanillin zusätzlich auftretenden Anregungsmaxima für die einzelnen untersuchten Steroide angegeben. VanillinSchwefelsäure-Reagenz dient als Sprühreagenz zur Darstellung von Steroiden auf Dünnschichtchromatogrammen und erzeugt mit zahlreichen verschiedenen Steroiden bei Bestrahlung mit einer langwelligen UVLampe sehr charakteristische und differenzierte Farbtöne. Die Substanz soll in ihrer Eigenschaft als Assoziationspartner gegenüber den Fluoreszenzchromogenen der Steroide geprüft werden: $0,2 \mu \mathrm{Mol}$ des Steroids werden in Gegenwart von $60 \mu \mathrm{g}$ Vanillin nach der angegebenen Schwefelsäure-Standardmethode behandelt. Auf Intensitätsangaben wurde verzichtet, da mit der Substanz unkontrollierbare Mengen von Fluoreszenzlöschern zugesetzt werden. Die angegebenen op- 
Tab. 1

Optische Daten der Fluoreszenzchromogene einzelner Steroide

\begin{tabular}{|c|c|c|c|c|c|c|}
\hline Trivialname & $\begin{array}{l}\text { Steroid: } \\
\text { Systematischer Name }\end{array}$ & $\begin{array}{l}\text { Anregungs- } \\
\text { maximum } \\
{[\mathrm{m} \mu]}\end{array}$ & $\begin{array}{l}\text { Standard- } \\
\text { abweichung } \\
{[ \pm \mathrm{m} \mu]}\end{array}$ & $\begin{array}{l}\text { Fluoreszenz- } \\
\text { maximum } \\
{[\mathrm{m} \mu]}\end{array}$ & $\begin{array}{c}\text { Standard- } \\
\text { abweichung } \\
{[ \pm \mathrm{m} \mu]}\end{array}$ & $\begin{array}{l}\text { Intensität } \\
\text { [Skt] }\end{array}$ \\
\hline \multicolumn{7}{|l|}{$\mathrm{C}_{18}$-Steroide } \\
\hline 1. Östron & 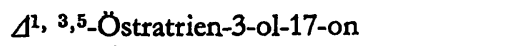 & 463 & 3 & 479 & 1 & 855 \\
\hline 2. Östradiol & $\Delta^{1}, 3,5$-Ostratrien-3, $17 \beta$-diol & 461 & 2 & 480 & 1 & 1150 \\
\hline 3. Óstriol & $\Delta^{1,}, 3,5$-Östratrien-3, $16 \alpha-17 \beta$-triol & 466 & 1 & 522 & 2 & 850 \\
\hline \multicolumn{7}{|l|}{$\mathrm{C}_{19}$-Steroide } \\
\hline 4. Androstandion & Androstan-3,17-dion & 477 & 1 & 504 & 3 & 13,0 \\
\hline 5. Androstendion & $\Delta^{4}$-Androsten-3,17-dion & 483 & 2 & 543 & 1 & 14,5 \\
\hline 6. Testosteron & $\Delta^{4}$-Androsten-17 $\beta$-ol-3-on & 470 & 1 & 543 & 3 & 36 \\
\hline 7. Androstandiol & Androstan-3 $\alpha, 17 \beta$-diol & 476 & 1 & 496 & 2 & 83 \\
\hline \multicolumn{7}{|l|}{$\mathrm{C}_{21}$-Steroide } \\
\hline 8. Pregnandiol & Pregnan-3 $\alpha, 20 \alpha$-diol & 482 & 2 & 523 & 3 & 150 \\
\hline 9. Progesteron & $\Delta^{4}$-Pregnen-3,20-dion & 469 & 1 & 525 & 1 & 40 \\
\hline 10. Pregnenolon & $\Delta^{5}$-Pregnen-3 $\beta$-ol,20-on & 496 & 2 & 525 & 1 & 95 \\
\hline 11. Cortexon & $\Delta^{4}$-Pregnen-21-ol-3,20-dion & 472 & 1 & 538 & 2 & 126 \\
\hline 12. Corticosteron & $\Delta^{4}$-Pregnen-11 $\beta, 21$-diol-3,20-dion & 472 & 1 & 538 & 2 & 284 \\
\hline 13. Aldosteron & $\Delta^{4}$-Pregnen-18-al-11 $\beta, 21$-diol-3,20-dion & 474 & 2 & 545 & 3 & 24 \\
\hline 14. Cortexolon & $\Delta^{4}$-Pregnen-17 $\alpha, 21$-diol-3,20-dion & 477 & 2 & 540 & 2 & 85 \\
\hline 15. Cortisol & $\Delta^{4}$-Pregnen-11 $\beta-17 \alpha, 21$-triol-3,20-trion & 474 & 1 & 541 & 2 & 245 \\
\hline 16. Cortison & $\Delta^{4}$-Pregnen-17 $\alpha, 21$-diol-3,11,20-trion & 473 & 1 & 540 & 1 & 109 \\
\hline \multicolumn{7}{|l|}{ 17. Tetrahydro- } \\
\hline cortison & Pregnan-3 $\beta, 17 \alpha, 21$-triol-11,20-dion & 471 & 1 & 543 & 2 & 65 \\
\hline 18. $\alpha$-Cortolon. & Pregnan-3 $\alpha, 17 \alpha, 20,21$-tetrol-11-on & 470 & 2 & 540 & 1 & 73 \\
\hline 19. $\beta$-Cortolon & Pregnan-3 $\alpha, 17 \alpha, 20,21$-tetrol-11-on & 474 & 3 & 540 & 1 & 73 \\
\hline 20. $\alpha$-Cortolol & Pregnan-3 $\alpha, 11 \beta, 17 \alpha, 20,21$-pentol & 495 & 2 & 537 & 3 & 125 \\
\hline 21. $\beta$-Cortolol & Pregnan-3 $\alpha, 11 \beta, 17 \alpha, 20,21$-pentol & 495 & 1 & 540 & 2 & 140 \\
\hline
\end{tabular}

Tab. 2

Spektrale Lage der in Gegenwart von Vanillin zusätzlich auftretenden Anregungsmaxima

\begin{tabular}{lc}
\hline Steroid $\left.^{2}\right)$ & 2. Anregungsmaximum in $\mathrm{m} \mu$ \\
\hline Ostron & 406 \\
Ostradiol & 430 \\
Östriol & 409 \\
Testosteron & 409 \\
$\alpha$-Cortolol & 408 \\
$\beta$-Cortolol & 406 \\
Cortisol & 401 \\
Cortisosteron & 403 \\
Cortexon & 404 \\
Cortexolon & 406 \\
\hline
\end{tabular}

2) Erklärung der Trivialnamen s. Tabelle 1.

\section{Tab. 3}

Fluoreszenzintensitäten in Skalenteilen (Skt) von $0,2 \mu \mathrm{Mol}$ Cortexon in Abhängigkeit von der Konzentration zugesetzter Carbonylverbindungen in $\mu$ Molen. (Werte in Skalenteilen)

\begin{tabular}{lcccccccccc}
$\begin{array}{l}\text { Carbonylver- } \\
\text { bindung }\end{array}$ & $\mu \mathrm{Mol}$ & 0,1 & 0,3 & 0,5 & 0,6 & 0,8 & 1,2 & 1,4 & 1,8 & 2,0 \\
\hline $\begin{array}{l}\text { Benzil } \\
\text { p-Dimethyl- } \\
\text { aminobenzalde- } \\
\text { hyd }\end{array}$ & 32,5 & 36 & 34 & 34 & 40 & 40 & 41 & 44,533 \\
\hline & 53 & 49 & 20,5 & 28,5 & 36,5 & 30,5 & 29 & 31 & 27,5 \\
\hline Vanillin & $\mu \mathrm{Mol}$ & 0,05 & 0,1 & 0,2 & 0,4 & 0,6 & 0,8 & 1,2 & \\
\hline
\end{tabular}

tischen Daten stellen Mittelwerte für die Anregungsmaxima aus 3 Messungen dar. Die Maxima für das Fluoreszenzlicht bleiben innerhalb der in Tabelle 1 aufgeführten Schwankungsbreite für die einzelnen Substanzen unverändert. Lichtquelle: Xenonlampe XBO $450 \mathrm{~W}+$ UV-durchlässiges Filter (Maximum bei-395 $\mathrm{m} \mu$ ).

\section{Abbängigkeit der Fluoreszenqintensität vom Molverbältnis zugesetzter aromatischer Carbonylverbindungen}

In Tábelle 3 sind Ergebnisse von Versuchen wiedergegeben, bei denen die Intensität des Fluoreszenzlichtes von Cortexon in Abhängigkeit von der Konzentration der "Carbonylverbindungen" p-Dimethylaminobenzaldehyd, Vanillin und Benzil gemessen wurde. Cortexon ist lediglich ein besonders typisches Beispiel für weiter untersuchte Kombinationen: Androstendion + Vanillin, Testosteron + Vanillin, Cortisol + Vanillin und Cortisol + p-Dimethylaminobenzaldehyd. In allen Fällen zeigten sich deutliche Maxima der Intensität bei bestimmten Molverhältnissen von Steroid zu Carbonylverbindung. Die Werte in Tabelle 3 beziehen sich auf $0,2 \mu \mathrm{Mol}$ Steroid, die zusammen mit der angegebenen Menge der Carbonylverbindung nach der Schwefelsäure-Standardmethode behandelt wurden. Anșchließend wurden die Intensitäten bei den jeweiligen Monochromatoreinstellungen für das Spektrum des reinen Steroids gemessen (Ab- 
lesegenauigkeit: 0,2 Skalenteile). Lichtquelle: Xenonlampe XBO $450 \mathrm{~W}+$ UV-durchlässiges Filter (Maximum bei $395 \mathrm{~m} \mu$ ).

Der Gang der Konzentrationsabhängigkeit ist bei Wiederholung der Meßreihe reproduzierbar. Die Carbonylverbindungen selbst haben in dem in Frage kommenden Spektralbereich keine meßbare Fluoreszenzintensität.

\section{Diskussion}

\section{Praktische Anwendungen der Fluorometrie}

Die in Tabelle 1 zusammengefaßten spektroskopischen Daten zeigen, daß sowohl die Anregungs- als auch die Fluoreszenzmaxima für einzelne Steroide hinsichtlich ihrer Lage und Intensität charakteristisch sind. Für eine spezifische Bestimmung einzelner Steroidhormone und ihrer Stoffwechselprodukte ist es von Vorteil, die spektroskopischen Eigenschaften ihrer Fluoreszenzchromogene in konz. Schwefelsäure zu kennen. Die optischen Daten der Tabelle 1 können daher als Grundlage zur Ausarbeitung spezifischer Bestimmungsmethoden für einzelne Steroide mit dem Doppelmonochromatorgerät herangezogen werden. Eine weitere Steigerung der Spezifität in der fluorometrischen Steroidanalyse war bisher dadurch verhindert, daB die Anregung der Fluoreszenz mit einzelnen Linien der Quecksilberlampe erfolgte, die durch monochromatische Filter isoliert werden können. Damit blieb die Spezifität im Bereich der Anregung auf eine kleine Anzahl von Linien beschränkt. Wir sind allerdings im Verlaufe unserer Untersuchungen zu der Auffassung gelangt, da $\beta$ die in den Tabellen 1-3 angegebenen fluoreszenzspektroskopischen Daten ausschließlich für die von uns gewählte apparative Anordnung, die in der Konstruktion des Zeiss'schen DoppelmonochromatorGerätes begründet liegt, Gültigkeit besitzen. Es handelt sich also um rein empirische Daten, die zwar bei Verwendung der gleichen Apparatur stets gut reproduzierbar sind, jedoch keinen Aufschluß geben über die wahre Größe der mit der Fluorseszenzemission einhergehenden Veränderungen der molekularen Quantenzustände.

Die spektrale Lage der Anregungs- und Fluoreszenzmaxima kann durch folgende in der Meßanordnung begründete Faktoren beeinflüßt werden:

\section{Spektrale Intensitätsverteilung der Lichtquelle}

2. Verlauf der Absorptionskurve eines vor die Lichtquelle geschalteten Filters

3. Verlauf der Absorptionskurve des Küvetteninhaltes (Lösungsmittel usw.), Absorptionen des Fluoreszenzchromogens außerhalb des Anregungsmaximums.

Auf Grund dieser therlegungen darf vor allem der Verlauf der Anregungskurve eines in Lösung untersuchten Fluoreszenzchromogens im wesentlichen als eine Resultierende aus den unter 1.-3. genannten Faktoren mit den speziellen Anregungsbedingungen des Fluores- zenzchromogens selbst angesehen werden. Diese Auffassung läßt sich durch folgende Beobachtungen belegen:

a) Mit der unveränderten Intensitätsverteilung der Xenonlampe, d.h. bei Entfernen des UV-durchlässigen Filters vor der Lichtquelle, können im Bereich $440-480 \mathrm{~m} \mu$ für die einzelnen Steroide bzw. Steroidgruppen charakteristische Anregungsmaxima gemessen werden (s. Tabelle 1).

b) Bei Einschalten des UV-durchlässigen Filters (Absorptionsmaximum bei $395 \mathrm{~m} \mu$ ) messen wir bei Zusatz von Vanillin im Bereich $400-430 \mathrm{~m} \mu$ ein zusätzliches Anregungsmaximum, dessen spektrale Lage bei den einzelnen Steroiden deutliche Unterschiede zeigt (s. Tabelle 2), während die Anregungsmaxima im Bereich $450-480 \mathrm{~m} \mu$ nur flach und wenig differenziert sind.

c) Bei Verwendung einer Wolframfaden-Glühlampe (Emissionsmaximum bei $540 \mathrm{~m} \mu$ ) erhalten wir wenig charakteristische Anregungsmaxima um $490 \mathrm{~m} \mu$.

Die Frage, ob die Anregungsspektren für die einzelnen Steroide verschieden sind, war bisher ungeklärt. In der vorliegenden Arbeit konnte diese Verschiedenheit zumindest für einzelne Gruppen von Steroiden - festgestellt werden. Die in Tabelle 1 mitgeteilten Daten sind rein empirische Werte. Dies hindert jedoch nicht ihre analytische Verwendbarkeit beim Arbeiten mit dem benutzten Doppelmonochromatorgerät. $\mathrm{Da}$ die Fluoreszenzchromogene sämtlicher Steroide unter genauen Standardbedingungen erzeugt wurden, geben die in Tabelle 1 angegebenen Intensitäten Aufschluß über die zu erwartende Empfindlichkeit. Die Individualität der Fluoreszenz- und Anregungsspektren hinsichtlich ihrer Lage und Intensität ist bei den hier untersuchten Steroiden so weit ausgeprägt, daß man - unter genauer Einhaltung der Standardbedingungen - die fluoreszenz-optischen Daten zusätzlich zu den optischen Daten der Lichtabsorption im sichtbaren und UV-Gebiet, die in einem Arbeitsgang in derselben Küvette gemessen werden können, zur Identifizierung von $\mu \mathrm{g}$-Mengen eines Steroids mit heranziehen kann. Die Fluoreszenzspektroskopie hat gegenüber der IR-Spektroskopie den Vorteil, von einem extremen Reinheitsgrad des Steroids unabhängig zu sein. Wegen des rein empirischen Charakters der fluoreszenz-optischen Daten eignet sich diese Methode lediglich zur Identifizierung bei Vergleich mit bekannten Steroiden. Bei der Schwefelsäuremethode sind die Spektren der Fluoreszenzchromogene allerdings von der Einhaltung strenger Standardbedingungen und in hohem Maße von der Reaktionszeit abhängig.

\section{Zur Struktur der Steroid-Fluoreszenzcbromogene}

Bei den hier untersuchten Steroiden wurde die für sehr viele Fluoreszenzerscheinungen im kondensierten Zustand charakteristische Stokes'sche Verschiebung in allen Fällen beobachtet. Durch die Reaktion der Schwefel- 
säure mit dem Steroid entstehen also Fluoreszenzchromophore, in denen ein strahlungsloser Utbergang des Elektrons von einem durch die Anregung erreichten oberen Term $T_{2}$ auf einen unteren $T_{1}$ möglich ist, von welchem aus der Rücksprung in den Grundzustand unter Lichtemission erfolgt. Wie im folgenden noch ausgeführt wird, entstehen bei der Einwirkung von Schwefelsäure auf das Steroid polare Strukturen vom Typ der Oniumsalze. Man kann also die ganze Erscheinung rein phänomenologisch als Fluoreszenzhalochromie auffassen; das chromophore System wird nämlich in größtem Ausmaß durch die Salzbildung verändert. Wie an Hand der Tabelle 1 deutlich wird, ist die Größe der Stokes'schen Verschiebung bei den einzelnen Steroiden sehr verschieden. Bei den $\mathrm{C}_{1^{-}}{ }^{-}$ Steroiden mit aromatischem Ring $\mathrm{A}$ ist sie besonders gering (s. Tabelle $1, \mathrm{Nr} .1-3$ ), ebenso bei den $\mathrm{C}_{19^{-}}$

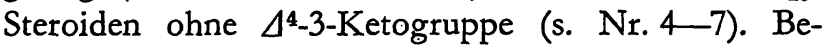
sonders groß ist sie bei den $\mathrm{C}_{21}$-Steroiden (Nr. 8-21). Die Größe der Stokes'schen Verschiebung steht also in einer gewissen Relation zum Typ der Steroidgerüste. Auch ein Vergleich der in Tabelle 1 angegebenen Intensitäten zeigt, daß die Intensitäten der Einzelverbindungen der 3 Steroidgruppen innerhalb eines bestimmten Bereiches liegen, ohne $\mathrm{da} B$ nennenswerte Überschneidungen festzustellen sind. - Als einzelne Fluoreszenzchromophore kommen in Betracht:

1. $>\mathrm{CHOH}:$ z. B. bei Androstandiol, $\alpha$-Cortolol, $\beta$-Cortolol

2. \C-OH: z. B. Östrogene

\section{3. $>\mathrm{C}=\mathrm{O}: \mathrm{z}$. B. Androstandion}

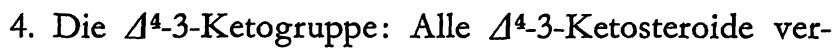
lieren die für zahlreiche indifferente Lösungsmittel charakteristische UV-Absorption bei $240 \mathrm{~m} \mu$.

Die Absorption des Anregungslichtes in konz. Schwefelsäure wird in den längerwelligen Bereich verschoben.

Wie aus Tabelle 1 deutlich wird, ist das Fluoreszenzvermögen einzelner Sauerstoff-Funktionen der Steroide bei konstanter Säurekonzentration sehr unterschiedlich. Als Extreme fallen besonders die starke Fluoreszenz der phenolischen $\mathrm{OH}-\mathrm{Gruppe}$ am Östrangerüst und die relativ schwache der sekundären Alkohol-Gruppen des Androstandiols auf. $\mathrm{Da}$ bei konstanter Protonenkonzentration des Reaktionsmediums derartig unterschiedliche Intensitäten auftreten und da die Protonisierbarkeit der verschiedenen Sauerstoff-Funktionen sicher verschieden ist, so kann man daraus schließen, $\mathrm{da} ß$ diese in ihrer protonisierten Form fluoreszenzchromophore Gruppen darstellen. - Steroide ohne Sauerstoff-Funktionen bilden in konz. Schwefelsäure keine UV-Chromogene (11). Da nach eigenen Erfahrungen alle untersuchten Fluoresżenzchromogene auch UV-Chromogene waren, ist die Schlußfolgerung berechtigt, daß sauerstoffreie Steroide keine Fluoreszenzeigenschaften in konz. Schwefelsäure besitzen. Lage und Intensität der Fluoreszenzspektren ändern sich mit der Konzentration der Schwefelsäure und der Reaktionstemperatur erheblich; wie dies vor allem von KaLANT (9) gezeigt wurde und von uns bestätigt werden konnte.

Dieses unter den verschiedenen Protonisierungsbedingungen typische Verhalten darf als ausreichender Hinweis für die Bildung von Oniumsalzstrukturen aus Steroiden in konz. Schwefelsäure gelten. Diese Auffassung kanin weiterhin gestützt werden durch die in den Tabellen 2 und 3 wiedergegebenen Messungen in Gegenwart von Substanzen, von denen bekannt ist, $\mathrm{da} ß$ sie in konz. Schwefelsäure Oniumsalze bilden und die somit Assoziationsgleichgewichte mit den SteroidFluoreszenzchromogenen aușbilden können. Die Ergebnisse der Messungen bestätigen die Annahme einer Assoziation zwischen dem Steroid und den Oniumstrukturen der Carbonylverbindungen. Damit wird die Existenz fluoreszenzfähiger Oniumstrukturen des Steroids wahrscheinlich gemacht: Wie man aus Tabelle 3 erkennt, ist die Wirkung der untersuchten Substanzen Vanillin, p-Dimethylaminobenzaldehyd und Benzil, denen lediglich die Carbonylfunktion am aromatischen Kern gemeinsam ist, gleichsinnig (Ausbildung eines Intensitätsmaximums).

Im Falle des Vanillins haben wir zusätzlich den Einfluß dieser Substanz auf die spektrale Lage der Anregungsund Fluoreszenzmaxima untersucht und die in Tabelle 2 angegebenen zweiten Anregungsmaxima gefunden. Es ist unwahrscheinlich, daß es sich hierbei um unspezifische, durch die Meßanordnung bedingte, Faktoren handelt, da die in Tabelle 2 angegebenen Anregungsmaxima konzentrationsabhängig sind und ihre Intensität mit steigender Vanillinkonzentration abnimmt, ein Konzentrations-Löscheffekt, wie er für die Fluoreszenz in Lösungen typisch ist. Die Lage der Fluoreszenzmaxima ist in Gegenwart von Vanillin nicht signifikant verändert. Dies zeigt, daß die Annahme einer Assoziationsverbindung zwischen den Oniumsalzen von Steroid und Vanillin die Verhältnisse am besten wiedergibt, da eine tiefgreifende chemische Veränderung des Steroidmoleküls offenbar nicht stattfindet.

Die in Tabelle 2 dargestellten Veränderungen sind sehr wahrscheịnlich die Erklärung für die experimentelle Erfahrung, daß bei Zusatz von aromatischen Aldehyden zum Schwefelsäure-Sprühreagenz die verschiedenen Steroide sich auf Dünnschichtchromatogrammen in sehr differenzierten Fluoreszenzfarbtönen darstellen lassen. Die Erscheinung läßt sich am einfachsten so deuten, daß der bei Bestrahlung mit polychromatischem UV-Licht sichtbar werdende Farbton als Mischfarbe des eigentlichen Fluoreszenzfarbtons mit dem an der Dünnschichtplatte reflektierten Anregungslicht aufzufassen ist, das nach Absorption der Anregungswellenlänge in der hierzu komplementären Farbe erscheint. 
Da die Lage des Anregungsmaximums in Gegenwart von Vanillin verändert wird, ergibt sich eine entsprechend veränderte Komplementärfarbe für das reflektierte Anregungslicht, die auch den Mischfarbton verändert.

Die Ausbildung von Intensitätsmaxima der Fluoreszenz bei Zusatz steigender Konzentrationen von aromatischen Carbonylverbindungen ist unter dem Aspekt der Assoziationsgleichgewichte theoretisch nicht $\mathrm{zu}$ erwarten. Die Intensitäten sollten vielmehr nach Erreichen eines Maximalwertes mit steigender Konzentration nicht wieder abfallen. Die Carbonylverbindungen lösen sich jedoch mit schwach gelber Farbe in konz. Schwefelsäure, so daß die Eigenabsorption der überschüssigen, nicht assoziierten Molekeln die Fluoreszenz um so stärker löscht, je größer ihre Anzahl im Lichtweg der Fluoreszenzstrahlung ist.

Die Ausbildung der Intensitätsmaxima sowie das Auftreten eines 2. Anregungsmaximums in Gegenwart von Vanillin sind als ein deutlicher Hinweis dafür zu werten, daß oberhalb eines bestimmten Konzentrationsverhältnisses von Steroid zu Carbonylverbindung die Carbonylverbindung in 2 Formen existiert, die sich durch ihre Lichtabsorption unterscheiden. Die eine Form wäre nach unserer Auffassung als das mit dem Steroidchromogen assoziierte, die andere als das freie Oniumsalz anzusprechen.

Die aus den Messungen erhaltenen Hinweise auf eine Assoziation der Oniumstrukturen legen die Annahme nahe, daß die Steroid-Fluoreszenzchromogene" auch unter sich, also ohne die Carbonylverbindungen, assoziiert sein können. Die in Frage kommenden Gleichgewichte dürften wegen der geringen Polaritätsunterschiede leicht beeinflußbar sein. Hierin wäre eine wesentliche Ursache der großen Variabilität der spektralen Eigenschaften dieser Fluoreszenzchromogene und damit der schlechten Reproduzierbarkeit der Schwefelsäuremethode zu suchen. Eine solche Assoziation wird auch von KALANT (9) angenommen.

Für die Struktur dieser Assoziationsverbindungen sind folgende Typen am wahrscheinlichsten:

Mögliche Onium-bə2n. Carbeniumstrukturen: a)<smiles>C=C(C)[OH2+]</smiles>

b)<smiles>[R]C([2H])[OH2+]</smiles>

c)<smiles>[R1][C]([R1])O</smiles>

Wabrscheinlichste Kombinationsmöglicbkeiten:

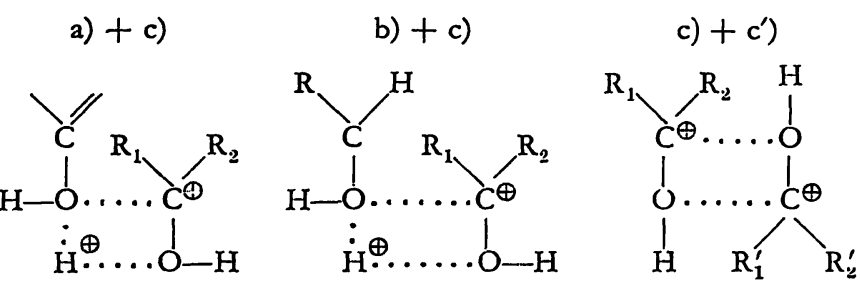

\section{Literatur}

1. Bierry, H. und B. Gouzon, C. R. Soc. Biol., Paris 122, 147 (1936). - 2. Umberger, E. J. und J. M. Curtis, J. biol. Chemistry 178, 275 (1949). - 3. BATES, R. W. und H. CohEN, Endocrinology 47, 166 (1950); 47, 182 (1950). - 4. Braunsberg, H., J. Endocr. 8, 11 (1952). - 5. GoldzIEHER, J. W.; J. M. BodenCHuK und D. Nolan, J. biol. Chemistry 199, 621 (1952). 6. Diczfalusy, E., Acta endocr., K'hvn 12, Suppl. XII., 1953.
7. Sweat, M. L., Analytic. Chem. 26, 773 (1954). - 8. Kalant, H., Biochem. J. 69, 93 (1958). - 9. Kalant, H., Biochem. J. 69, 79 (1958). - 10. Strickler, H. S., R. C. Grauer und M. R. Caughey, Analytic. Chem. 28, 1240 (1956). - 11. NowaCZYnskr, W. und P. R. Steyermark, Canad. J. Biochem. Physiol. 34, 592 (1956).
Professor Dr. Hj. Staudinger Physiologisch-Chemisches Institut der Justus Liebig-Universität Gießen 63 Gießen, Friedrichstr. 24 